\title{
Detecting Emerging Activity-Based Working Traits through Wearable Technology
}

\author{
ALESSANDRO MONTANARI, Computer Laboratory, University of Cambridge \\ CECILIA MASCOLO, Computer Laboratory, University of Cambridge \\ KERSTIN SAILER, Space Syntax Laboratory, University College London \\ SARFRAZ NAWAZ, Computer Laboratory, University of Cambridge
}

\begin{abstract}
A recent trend in corporate real-estate is Activity-Based Working (ABW). The ABW concept removes designated desks but offers different work settings designed to support typical work activities. In this context there is still a need for objective data to understand the implications of these design decisions. We aim to contribute by using automated data collection to study how ABW's principles impact office usage and dynamics.

To this aim we analyse team dynamics and employees' tie strength in relation to space usage and organisational hierarchy using data collected with wearable devices in a company adopting ABW principles. Our findings show that the office fosters interactions across team boundaries and among the lower levels of the hierarchy suggesting a strong lateral communication. Employees also tend to have low space exploration on a daily basis which is instead more prevalent during an average week and strong social clusters seem to be resisting the ABW principles of space dynamics. With the availability of two additional data sets about social encounters in traditional offices we highlight traits emerging from the application of ABW's principles. In particular, we observe how the absence of designated desks might be responsible for more rapid dynamics inside the office.

In more general terms, this work opens the door to new and scalable technology-based methodologies to study dynamic office usage and social interactions.
\end{abstract}

ACM Reference format:

Alessandro Montanari, Cecilia Mascolo, Kerstin Sailer, and Sarfraz Nawaz. 2018. Detecting Emerging Activity-Based Working Traits through Wearable Technology. 1, 1, Article 1 (February 2018), 24 pages.

https://doi.org/10.1145/nnnnnnn.nnnnnnn

\section{INTRODUCTION}

The study of the impact of space and human interaction in the workplace has been the subject of research for many years. Several studies have analysed the relationship between physical office space and interaction patterns, as well as innovation and performance [29, 42-44]. The common facet of these studies is that they considered traditional offices where employees have assigned desks and static routines. However, recently, several design principles have been emerging with the objective of realizing dynamic and agile working environments that can better support knowledge workers.

The Activity-Based Working (ABW) concept is one of these principles. It aims at architecting the office based on the activities the employees have to perform daily [4]. At the foundation of ABW there is the freedom for employees to chose where and when they work. This translates into absence of allocated desks with the assumption

Permission to make digital or hard copies of all or part of this work for personal or classroom use is granted without fee provided that copies are not made or distributed for profit or commercial advantage and that copies bear this notice and the full citation on the first page. Copyrights for components of this work owned by others than ACM must be honored. Abstracting with credit is permitted. To copy otherwise, or republish, to post on servers or to redistribute to lists, requires prior specific permission and/or a fee. Request permissions from permissions@acm.org.

(c) 2018 Association for Computing Machinery.

XXXX-XXXX/2018/2-ART1 \$15.00

https://doi.org/10.1145/nnnnnnn.nnnnnnn

, Vol. 1, No. 1, Article 1. Publication date: February 2018. 
that employees will move within the office by choosing the best functional work setting for the tasks to complete and that best matches their preferences, and thus improving productivity. As a side effect, $\mathrm{ABW}$ generally reduces costs due to a lower requirement on total floor space [16]. It is also likely to increase communication between groups and foster knowledge sharing and collaboration given the mobility that derives from having unallocated desks. Even if ABW is not a new concept, its adoption has recently been raising. However its benefits are not yet well understood $[4,53]$. Some works have analysed ABW offices using traditional ethnographic methods of participant observations and surveys to study productivity, health and satisfaction $[4,34,38]$ or provided a theoretical model of the benefits and risks of ABW [53].

Observing agile working through traditional methods (such as surveys, or participant observations) requires considerable effort, given the high mobility and dynamics of the setting. This is evidenced by the limited number of existing research studies on ABW offices. A typical approach is for instance to investigate patterns of occupancy and space usage [46], which are then averaged across teams. With individuals enjoying free choice of where to work for any given point in time, occupancy patterns of teams would be almost impossible to track. Another relevant research insight - the distance dependency of frequent interaction $[3,44,45]$ would be impossible to repeat in an ABW environment with traditional research methods, since fixed desk locations in traditional offices are typically used to calculate distances between co-workers. In contrast, non-assigned desks lead to constantly changing patterns of proximity and co-presence, since members of staff sit next to different people all the time.

In this work we focus on and analyse two core aspects of $A B W$, flexible use of office space and collaboration opportunities, relying on data automatically collected through wearable devices. The work, which exploits the advantages of technology in automating the collection of fine grained temporal data of a number of individuals, shows how our methodology is able to detect behavioural traits and relate them to $A B W$ core principles. We have exploited a solution based on Bluetooth Low Energy (BLE) and 3-axis accelerometers [39], which has been deployed together with other 17 static beacons in a company office. We captured a data set of close proximity contacts, location traces and physical activity (walking) of 25 employees working in a flexible and dynamic office for a period of 4 weeks. The office has been intentionally designed with ABW principles in mind and offers the employees several opportunities for adopting flexible working practices. This study allowed us to investigate social ties in relationship to the hierarchy and roles of employees and their use of office space in a specific kind of work environment that has not been thoroughly studied before.

The specific contributions of this work are as follows:

- We show how, in this company, interactions easily cross team boundaries, in line with ABW principles. We also find that a good amount of these inter-team contacts happen in the kitchen and in circulation areas suggesting a more serendipitous nature than the ones happening at the workstations. However, the mix among different layers of the organizational hierarchy is not as strong, with more contacts among people in the lower levels. It could be hypothesised that $\mathrm{ABW}$ concepts might facilitate lateral communication (comparing with reports on non ABW studies).

- We show how the absence of assigned desks, at the core of ABW and flexible office spaces, relates to a good usage of different spaces in the building. This however emerges when we consider a larger temporal scale of an average working week while it is less prevalent within a day.

- We further find relevant spatial and locational effects in the data. Spatial preferences arise from strong contact ties: pairs of individuals connected with a strong tie (defined as a higher than average number of contacts taking place in locations away from desks) are more likely to choose to sit at the same workbench. This points towards a possible mismatch between $\mathrm{ABW}$ principles and the actual use of space where people seem to choose working spaces based on the presence of other colleagues at the same location rather than exclusively based on the task they have to complete. However, this might also be related to the nature of work done as team affiliation however played a strong role in this. 
- We discover differences in the temporal contact patterns between the company we studied and other more traditional offices by comparing our data set with two other ones. We discover how our participants have on average shorter contacts and how the network has the potential to better enables quicker communication of information (both in times and hops) compared with traditional offices. A potential effect of the ABW principles adopted.

In more general terms, we show that our methodology allows the study the effects of ABW principles applied in offices. This work goes one step further than previous work on ABW [26] in analysing not only occupancy data but also contacts between participants and by showing the potential of technology in gathering contacts data in a challenging environment where a traditional observation modality would struggle. The comparative analysis with more traditional offices also helps the understanding of the behavioural characteristics emerging from the application of ABW's principles.

\section{MOTIVATION AND RELATED WORK}

Understanding the communication and collaboration patterns of employees is critical for the efficient and effective operation of the organization and could lead to improvement in productivity and exchange of innovative ideas $[2,10,32,43,49]$. For this reason, in the field of architecture, increasing effort is put in the design of spaces that could potentially promote more frequent and serendipitous face-to-face contacts [3].

In recent years, the increase of knowledge-intensive firms led to the emergence of Activity-Based Working $(\mathrm{ABW})$ concepts to design offices that better support modern workforces. The concept of ABW is complex and each organization can adapt it to its specific needs and possibilities. However, three core principles are common to different implementations of the concept: (1) absence of allocated desks, (2) availability of diverse spaces and settings including those for concentration and collaboration and (3) allow interaction and collaboration to spread across team boundaries. The idea is that employees can choose the workstation that best matches the current task they have to complete and their personal preferences, possibly even switching between workstations during the day [4]. As result, usually, offices designed following ABW principles consist of a mix of different typologies of areas: isolated and quiet workstations for individual focused work, large and open settings where serendipitous interactions can flourish and meeting rooms for private discussions. In this paper we focus on these central principles and introduce a methodology based on technology and analysis techniques which is able to help in understanding the degree of effectiveness of these principles.

Activity-Based Working with unallocated desks is still an exception rather than the norm in corporate workplaces: a one-year study of working environments in 2016 showed that only $4 \%$ of the surveyed workplaces embraced ABW [34]. Given that this "agile" working style is on the rise however, our work takes a further step towards a better understanding of its impact over workplaces. Some previous work has analysed companies adopting ABW principles using traditional ethnographic methods of participant observations and surveys. In a recent study where more than 500 workplaces have been surveyed (with ABW and without), Leesman found that $\mathrm{ABW}$ environment deliver performance improvements only when the employees correctly embrace the central principle of mobility. However, most of the employees who work in an ABW office still keep habits typical of traditional workplaces and present rather static work styles [34]. Appel-Meulenbroek et al. surveyed and observed four organizations with ABW and similarly found that most of the people use up to two different types of spaces, never switch work location during an average day and concluded that the offices are not always used as intended [4]. By contrast, Meijer et al. focused on workers' health and productivity and found that ABW had some positive effects on general health in the long term [38]. With this work we aim at contributing to the increasing efforts in the study of this new kind of workplaces and work practices.

, Vol. 1, No. 1, Article 1. Publication date: February 2018. 


\subsection{Mobile Sensing for Office Analytics}

Automatic systems have been used to study human behaviour in the workplace. Brown et al. deployed RFID-based systems to collect face-to-face contacts to study how different cultures interact with others in different job roles and the impact of physical space on social interactions [7, 8]. Choudhury et al. first presented the Sociometer, a wearable device equipped with infrared sensors and a microphone to measure face-to-face contacts [14]. Olguín et al. continued the development and presented the SocioMetric badges which rely on similar sensors and have been employed in several organizations to study interaction patterns and people behaviour [40, 41]. Other deployments of the same technology investigated how social interactions can affect productivity [51] and how they relate to electronic communication [54]. Lepri et al. employed the SocioMetric badges to collect a multilayer dataset comprising different information sources (sensor data, surveys and experience sampling) about fifty-three employees of an Italian research centre [35]. Do et al. used the same dataset to develop a model to automatically discover and label social activities (e.g. coffee breaks and meetings) starting from social contacts and location information [20]. Also other technologies have been proposed, for instance, RFID [11], WiFi [37] and hybrid approaches with radio and ultrasound sensors [24].

Bluetooth Classic has often been used in contact tracking because of its ubiquity on consumer devices (i.e. smartphones) $[1,13,19,21,25,48]$. Clauset et al. analysed data collected with Bluetooth and highlighted how different structural patterns of social networks can be extracted by analysing data at different timescales and how this could lead to bias in the analysis [15]. More recently Bluetooth Low Energy (BLE) has also been adopted to collect data about human behaviour. Townsend et al. tested 4 different smartphones (2 Android and 2 iOS) to asses if they could detect each other using BLE [50]. Boonstra et al. deployed an Android and iOS app to 14 participants for a period of one week in a research institute to collect data about social contacts [5]. However, the authors offered a limited evaluation of their system by using only two meetings during the study period to validate their methodology and they did not collect location information about the participants. Other works instead have used simple wearable BLE tags, capable of transmitting only, to study mobility patterns of large gatherings [27, 28].

We evaluated the capabilities of BLE to monitor people proximity in the workplace finding it appropriate for office analytics achieving a considerable accuracy in discriminating between proximity and non-proximity. We also highlighted the limitations imposed on BLE parameters setting by two popular wearable platforms (i.e, Android Wear and Tizen OS) and their impact on power consumption [39]. In [39] we used a subset of collected data described here (people proximity contacts only) exclusively to evaluate BLE proximity detection power on custom and commercial devices (i.e., parameters tuning, power consumption and detection accuracy). In this paper we use also the location traces and we describe the study of the company dynamics and working style with focus on the impact of ABW principles.

While all of the above mentioned studies investigate settings with fixed desk assignments (e.g., research laboratories, call centers and banks), in this paper we focus on a very dynamic office where employees have flexibility in where, how and when they work. Employees can choose their preferred desk or alternative work point (such as benches, breakout spaces, meeting rooms etc.) every day and they can change settings during the day. The company also operates flexible hours and staff may work off site completely. All of this results in an unusually dynamic workplace environment. To the best of our knowledge, only one work used technology to study this kind of workplaces. Ianeva et al. used RFID tags embedded into employees' badges to monitor occupancy of spaces [26]. The authors found that three kinds of areas (cafeteria, private booths and meeting rooms) were consistently under-occupied, revealing a mismatch between intended and current use of these areas. With this work we go one step further in the analysis of ABW workplaces by including data about proximity contacts between employees. This allows us to study ABW principles concerning communication and collaboration and to link usage of space with interpersonal contacts. In Table 1 we offer a closer comparison with related works 
Table 1. Comparison between our work and previous work that adopted sensors to study office spaces.

\begin{tabular}{|c|c|c|c|}
\hline Paper & Context & Main Aspect Studied & Main Finding \\
\hline Wu et al. [54] & $\begin{array}{l}\text { Company with } \\
\text { assigned desks }\end{array}$ & $\begin{array}{l}\text { Effect of social structures on knowledge transfer } \\
\text { and productivity }\end{array}$ & $\begin{array}{l}\text { Network cohesion in face-to-face networks is positively correlated with higher productivity } \\
\text { in particular when executing complex tasks. }\end{array}$ \\
\hline Olguín and Pentland [40] & $\begin{array}{l}\text { Company with } \\
\text { assigned desks }\end{array}$ & Effect of physical activity and speech on job performance & Positive correlation between task completion time and physical activity and speech level \\
\hline Olguín et al. [41] & $\begin{array}{l}\text { Company with } \\
\text { assigned desks }\end{array}$ & Employees' job Satisfaction & $\begin{array}{l}\text { More communication leads to less satisfaction. } \\
\text { More central positions in the organisation leads to less satisfaction. }\end{array}$ \\
\hline Waber et al. [51] & $\begin{array}{l}\text { Company with } \\
\text { assigned desks }\end{array}$ & Effect of social groups on productivity & $\begin{array}{l}\text { Productive employees belong to the strongest social groups. } \\
\text { Achieve stronger social groups by changing breaks schedule. }\end{array}$ \\
\hline Brown et al. [8] & $\begin{array}{l}\text { Company with } \\
\text { assigned desks }\end{array}$ & Effect of spatial layout on social interactions & $\begin{array}{l}\text { Purposely designed offices can promote inter-team mixing. } \\
\text { Central food areas host many inter-team contacts. }\end{array}$ \\
\hline Brown et al. [7] & $\begin{array}{l}\text { Research institute } \\
\text { with assigned desks }\end{array}$ & $\begin{array}{l}\text { Effect of individuals' cultural differences and spatial layout } \\
\text { on serendipitous interactions }\end{array}$ & $\begin{array}{l}\text { Cultural dimensions (Power Distance, Individualism and Masculinity) affect the likelihood } \\
\text { of contacts between people with different roles. } \\
\text { Printers and kitchens are likely places for serendipitous inter-group contacts. }\end{array}$ \\
\hline Génois et al. [22] & $\begin{array}{l}\text { Company with } \\
\text { assigned desks }\end{array}$ & Analyse social contacts for epidemiology studies & $\begin{array}{l}\text { The spread of infectious diseases is hindered by the sparsity of the contacts. } \\
\text { Vaccinations of linkers between communities can prevent epidemic outbreaks. }\end{array}$ \\
\hline Ianeva et al. [26] & $\begin{array}{l}\text { Company adopting } \\
\text { ABW principles }\end{array}$ & $\begin{array}{l}\text { Monitor and understand the building occupancy rate } \\
\text { and use of shared areas }\end{array}$ & $\begin{array}{l}\text { Several office areas are under-occupied highlighting a miss match } \\
\text { between intended and actual use of the office }\end{array}$ \\
\hline Our work & $\begin{array}{l}\text { Company adopting } \\
\text { ABW principles }\end{array}$ & $\begin{array}{l}\text { Analyse core aspects of } \mathrm{ABW} \\
\text { and comparison with traditional offices }\end{array}$ & $\begin{array}{l}\text { High degree of inter-group contacts, especially in the lower levels of the hierarchy. } \\
\text { The principle of not having allocated desks seems not completely adopted. } \\
\text { Different network characteristics between traditional offices and offices adopting } \\
\text { ABW principles. }\end{array}$ \\
\hline
\end{tabular}

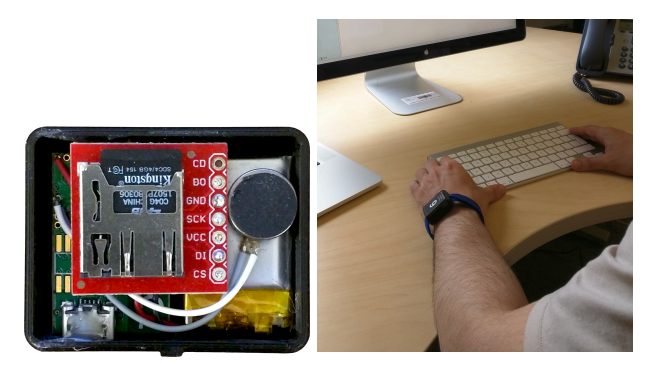

Fig. 1. Electronics (left) enclosed in 3D printed box $(3 \times 4 \times 1.5 \mathrm{~cm})$ and complete prototype attached to velcro wristband (right).

that not only used a similar data collection methodology but also studied environments similar to the one we considered (companies and research institutes) and conducted alike analysis (e.g. collaborations patterns and role of office space). We omit an in-depth comparison with other works that have studied completely different environments such as conferences, schools, museums and hospitals. The table highlights that we study a company that adopts ABW principles and has a dynamic working style, while the majority of previous work focused on traditional office spaces with allocated desks. Our aim with this work is to provide an understanding of people behaviour in this kind of companies. By contrast, most of previous work focused on the relation between social contacts and productivity.

\section{WEARABLE PLATFORM FOR PROXIMITY SENSING}

We now briefly describe the platform used [39] for the collection of the data on which this study is based.

We employed a developer board from Mbienlab Inc. which consists mainly of a BLE transceiver (Nordic's nRF51822) and a 3-axis accelerometer (Freescale MMA8452Q). The prototype also includes an SD card socket to log data about nearby BLE devices and a 100mAh 3.7V lithium battery rechargeable via micro USB. Figure 1 (left) shows the electronic components enclosed in a 3D printed box we designed. To allow the participants to wear the device on their wrist, the box permits to attach a velcro strap as shown in Figure 1 (right). 
Table 2. Mobility profiles we asked our participants to identify with. Source: Leesman's study 2016 [34].

\begin{tabular}{cl}
$\begin{array}{c}\text { Profile } \\
\text { Name }\end{array}$ & \multicolumn{1}{c}{ Mobility Profile } \\
\hline Profile 1 & $\begin{array}{l}\text { I perform most/all of my activities at a single work setting } \\
\text { and rarely use other locations within the office. }\end{array}$ \\
Profile 2 2 & $\begin{array}{l}\text { I perform the majority of my activities at a single work setting } \\
\text { but also use other locations within the office. }\end{array}$ \\
Profile 3 & $\begin{array}{l}\text { I perform some of my activities at a single work setting } \\
\text { but often use other locations within the office. }\end{array}$ \\
Profile 4 & $\begin{array}{l}\text { I use multiple work settings } \\
\text { and rarely base myself at a single location within the office. }\end{array}$ \\
\hline
\end{tabular}

\subsection{Data Collection}

Each device collects several pieces of information about other nearby BLE devices and about the participant wearing the device. For each device in the vicinity, it logs the MAC address, the Received Signal Strength (RSS) and the channel on which a packet from the other device has been received. The information is timestamped with the current time. The location information is provided by additional BLE devices (static beacons from now on) deployed in the building which are static and are associated with a certain area, usually a room or a desk. These devices continuously transmit a unique identifier of the area they are associated with, which is then used by the wearable devices to infer the current location.

The raw data from the accelerometer is processed on the device with a step detection algorithm [55] to detect whether the user wearing it is stationary or walking. The moment the user starts to walk, when he or she stops and the number of steps taken during the walk are then recorded on the SD card.

\section{WORKPLACE DEPLOYMENT}

The dataset we analyse in this work has been collected with our BLE platform in an architecture company in London (Spacelab Ltd.) which employs about 35 people. The company occupies a building which consists of two floors with a staircase opening in the middle (Figure 3). The two large open spaces host different workstations where several employees share the same large table. There are meeting rooms on both floors, while the kitchen and break out area are at the lower ground floor. The company adopts ABW principles and has a very dynamic and flexible working style. Employees do not have assigned desks, the work tasks are fluid and people have considerable interaction.

To understand how the participants perceived the mobility level of the workplace we administered a survey to the company employees where we asked to identify their mobility profile with one of the four used by the Leesman's study [34] of workplaces that adopt the Activity-Based Working style (reported in Table 2). We received 21 responses and we found that $38 \%$ of the participants identify themselves in the two profiles that describe more mobility (Profile 3 and 4) and only 10\% identified with Profile 1. Instead, in the Leesman's study only $27 \%$ of the employees identified themselves in Profile 3 and 4 and 32\% in Profile 1. This indicates that the working style in the company might be more dynamic compared to other more traditional offices. We also asked our participants to rate their agreement with the statement "I believe I move more at Spacelab than in other companies I worked" on a likert scale from 1 to 5, and $47.6 \%$ of the participants responded with "Strongly Agree", showing that this company might present different dynamics than other offices. Figure 2 reports the data collected in both parts of the survey. 

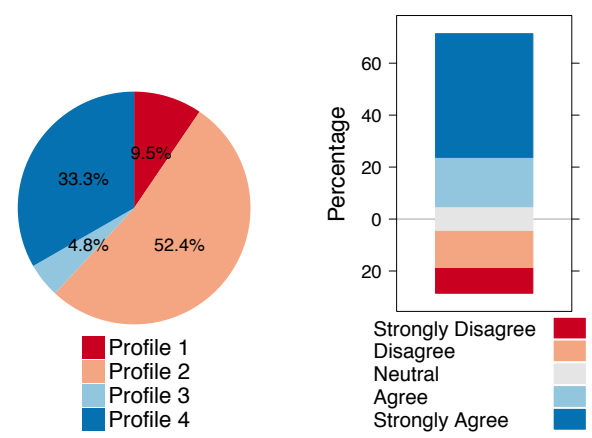

Fig. 2. Percentage of participants identified with each mobility profile (left) and agreement with the statement "I believe I move more at [blinded company] than in other companies I worked" (right).

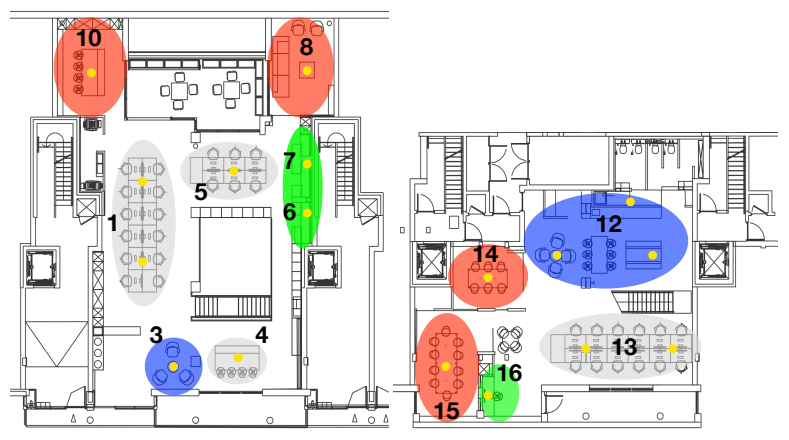

Fig. 3. Floor plans of the first floor (left) and lower ground floor (right) of the office space studied in this work. The yellow circles represent the location of the static BLE beacons. The coloured shadows represent the category of the locations.

Our study has been approved by the University of Cambridge Ethics Committee. Before the study all participants have been informed about its purposes and about the data that was being collected. The participants accepted to take part in the study voluntarily. The collected data have been anonymised at the source (i.e., there was no reference to the users in the data).

\subsection{Participants}

We recruited 25 participants (15 females) aged 21-44 $(\mu=31)$ for a period of four weeks between September and October 2015. The company is structured into five teams: Architecture (4 participants), Interior Design (10 participants), Workplace Consultancy (6 participants), Project Management (1 participant) and Administration (4 participants).

The vertical structure comprises 7 levels, from the top level (1), to the bottom (7). At the top of the hierarchy is one of the two Partners who works mainly with the Architecture team. The second highest level are Directors and the Project Manager (4 participants). The third and fourth levels consist respectively of Associates (2 participants) and Senior architects, designers and analysts (7 participants). At the fifth and sixth levels there are architects, 
designers and analysts (4 participants) and Assistants (3 participants). The Administration team was counted as a seventh level for consistency, although it would be fair to consider it as external to the hierarchical levels.

\subsection{Proximity Traces}

The raw data collected by the Bluetooth devices has to be processed in order to classify the contact events as proximity or not. We adopted a supervised machine learning approach where we trained a binary classifier with a set of examples labelled as "proximity" or "non-proximity". We were not interested in measuring the actual distance between the participants but only if they were close to each other as during a normal conversation.

To label the examples, we performed participant observations for three days during the study. During each observation, a researcher followed a person and annotated on a spreadsheet all the social interactions the person had. Only those interactions that happened in close proximity, i.e. up to a distance of 3 meters between people have been recorded by the researcher, given our interest in detecting fine grained proximity between people. For each interaction event the researcher recorded the start time, the end time, the location inside the office and the other people involved. A total number of 401 interactions among 18 different participants have been captured over a period of 19 hours.

Using only the data collected during the three days of participant observations we built a training set where the positive examples ("proximity" label) were labelled with the observed communication events. The negative examples instead ("non-proximity" label) were labelled using the static beacons. After computing which static beacon had the strongest signal strength (i.e. the closest one) at each point in time, we selected those periods where the participants were at different locations and we used them as "non-proximity" examples.

For each pair of individuals that have been observed we extract from their devices the stream of raw data relative to the other device (timestamped received packets with MAC address and RSSI value). We then merge the two streams into one in order to have more data for the classification. We then split this stream of data into non-overlapping one minute windows. For each window we compute the following features: median RSSI, min RSSI and max RSSI which, after several tests, are resulted to be the ones that perform better. When two people are very far from each other (e.g. in different floors of the building) the two devices will not receive any packet and this will result in missing values in the data set. In our context, those missing values are meaningful because they indicate that the two devices were not in range and we do not want a machine learning algorithm to ignore them. For this reason we replace the missing values with the value -110 which represents a very low RSS and it is below the minimum detectable power by our device $(-105 \mathrm{dBm})$. Once we have segmented the data streams for each pair of participants in 1-minute windows and computed the aforementioned features we aggregate all the windows (which for us represent single examples for the supervised machine learning algorithm) into a single data set. At this point we overlap the participant observations and we label each window with "proximity" or "non-proximity".

The resulting dataset presents class imbalance because for each pair of people we label the positive examples from the interaction events, which represents a limited period of the day, but we derive the negative examples from the times when they are at different locations in the building and these could cover longer periods of the same day. Thus we over-sample the minority class generating synthetic examples using the SMOTE technique [12] in order to balance the two classes.

For the classification we adopted Decision Trees (C4.5) and we evaluated them with stratified 10-fold crossvalidation. The implementation of the algorithm was taken from Weka version 3.7.13 [36] where we used all the default values for the parameters.

Table 3 reports the classification accuracy metrics divided by class and their average (area under the Receiver Operating Characteristic curve $=0.993$ ), showing how well the classifier is able to distinguish between the proximity and non-proximity cases. 
Table 3. Classification accuracy metrics by class.

\begin{tabular}{lccc} 
& TP Rate & FP Rate & F-Measure \\
\hline Non-proximity & 0.981 & 0.014 & 0.984 \\
Proximity & 0.986 & 0.019 & 0.984 \\
\hline Average & $\mathbf{0 . 9 8 4}$ & $\mathbf{0 . 0 1 6}$ & $\mathbf{0 . 9 8 4}$ \\
\hline
\end{tabular}

\subsection{Location Traces}

To collect location traces (at the desk level) we deployed seventeen BLE static beacons (Figure 3). Each desk in the building was covered by a beacon or two if the desk was too big. The beacons where configured to transmit packets 5 times a second with a range of about 4 meters.

To associate the current approximate location to the participants at each point in time, the data received from the static beacons was grouped into non-overlapping windows of 1 minute. We then computed the median value of the received signal strengths (RSS) from the different beacons. This process removes high frequency variations in the data which might ruin the location inference. At the end we chose the location for each time period by selecting the beacon with the strongest median RSS, which represents the closest one to the user. To improve the location estimation we used the accelerometer data. With the step detection algorithm [55] we know when the participants are walking and given that a person changes location only when she walks, we could remove spurious changes in location (which might be due to reflections in the radio signals) if the user was not walking at that time. Using the location traces we infer the desk each participant used each day by selecting the one where the person spent most of the time.

The different locations were grouped in 6 categories represented by the colours in Figure 3:

Open space workstations: shared workstations that can accommodate several people and represent the main areas where work is done in the company (colour grey).

Meeting rooms: four meeting rooms are present in the building, two on the first floor and two in the lower ground floor (colour red) and all of them have a table in the middle.

Private workstations: small areas where individual work (or maximum for two people) can be carried out (colour green).

Breakout areas: a relatively large open kitchen is present in the lower ground floor and a small table with magazines on the first floor (colour blue).

Circulation spaces: this is not an exact location because we did not deploy beacons in the space around desks. However, this label is used to tag contacts between people that are not close to the same static beacon. When this happens one of the participants is co-located with one beacon and the other participant with a different nearby beacon, in this case the contact between the two is tagged as happening in Circulation.

Outside office: the absence of location information while the device was in use is interpreted as if the user was outside.

This is a semantic organization of the static beacons that we will use later in the paper, however the spatial granularity of the location dataset is at the level of the single beacons.

\subsection{Metadata About the Study}

During the 4 -week long study we lost $10.8 \%$ of the total amount of data that we were expecting to collect due to failures. These failures are due to different causes: device malfunctions, device out of battery, device forgotten at home or lost ( 2 devices were lost). $30 \%$ of the data did not contain any contacts because, although the devices worked properly, they were not in use but they were charging at the charging station. This could be due to the 
fact that the working style is very dynamic and people are often outside to visit construction sites and probably forgot to wear the device.

We noticed that the devices have been mostly used during the first two weeks of the study, therefore we decided to consider only them for our analysis. From the collected raw data we extracted 2190 proximity contacts with a temporal and spatial resolution respectively of one minute and 5/6 meters [39]. We expect a lower spatial resolution, compared to the $3 \mathrm{~m}$ coarse distance estimate used during participants observations, because it is likely that the observer annotated contacts at a larger distance, particularly in certain situations (e.g. large group meeting). Additionally, reflections and different orientations of the device might result in "proximity" detections even if people were actually farther apart. This means that our approach is not suitable for accurate distance measurement, given the noisy RSSI values, but it provides reliable information about proximity between people which we use to study potential communication opportunities. We also extracted minute by minute traces of the locations visited during the two weeks to study people mobility.

\section{ANALYSIS OF ABW PRINCIPLES IMPACT}

\subsection{Organizational Structure and Interactions}

In this section we study how the organizational structure of the company relates to the contact patterns of the employees to identify the ABW impact. In particular, we examine two aspects of the organization: the horizontal structure where people are arranged into teams and the hierarchical vertical structure of who reports to whom. Previous work has highlighted the importance of interactions between members of different teams as a source of new ideas and a way to increase productivity $[2,10,32,43]$ and ABW principles are certainly based around these aims.

Beginning with the analysis of the horizontal structure, Figure 4(a) shows the normalized number of contacts for each pair of participants aggregated over the entire duration of the study. Contacts have been normalized by the number of days both participants were in the office at the same time (overlaid circles) in order to account for the fact that some people were in the office more than others. The ordering of the participants on the axis is such that adjacent participants belong to the same team. With this ordering, the contacts along the diagonal from bottom-left to top-right represent intra-team interactions. From Figure 4(a) we observe that different teams have a good amount of contacts with one another even if some of the strongest contacts are between members of the same team.

To better quantify the relation between inter and intra-team contacts we computed the number of these contacts for each team normalizing the results by the sizes of the groups involved to account for the number of possible pairs. Figure 5 shows the results. The intra-team contacts are obviously higher as expected when people work together but it is possible to see that the number of inter-team contacts is similar for all groups. We verified the similarity between the inter-team contacts for the four groups with the TOST Equivalence procedure using the Wilcoxon Rank-Sum Test [47, 52]. We found that the similarity is significant (p-value $<0.05)$ within $[-18,18]$ equivalence bounds for all pairs of groups except for Architecture and Workplace which is significant within [-25, 25] and Architecture and Admin within [-22,22] equivalence bounds. This workplace shows a large number of opportunities for interaction across teams (proximity contacts) which could be one result of the implementation of ABW's principles. Figure 4(a) appears almost randomly distributed, if compared to examples of traditional open-plan workspaces [44], where team clustering is much more prevalent.

To gain an insight about the nature of the inter-team contacts we looked at the total number of contacts happened at each location. We discovered that while most of the inter-team contacts happen at the Workstation \#1 (38\% of the contacts), the second and fourth locations for number of inter-team contacts are Circulation and Kitchen, respectively with $28 \%$ and $8 \%$ of the contacts. The open space workstations are the main locations where work is done in the company hence it is expected to see a high number of inter-team contacts. However, the 


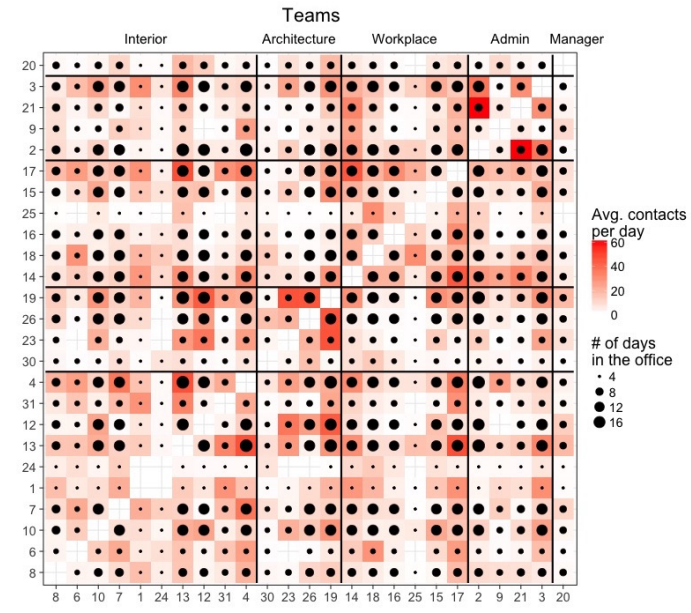

(a) Participants sorted by the team they belong to.

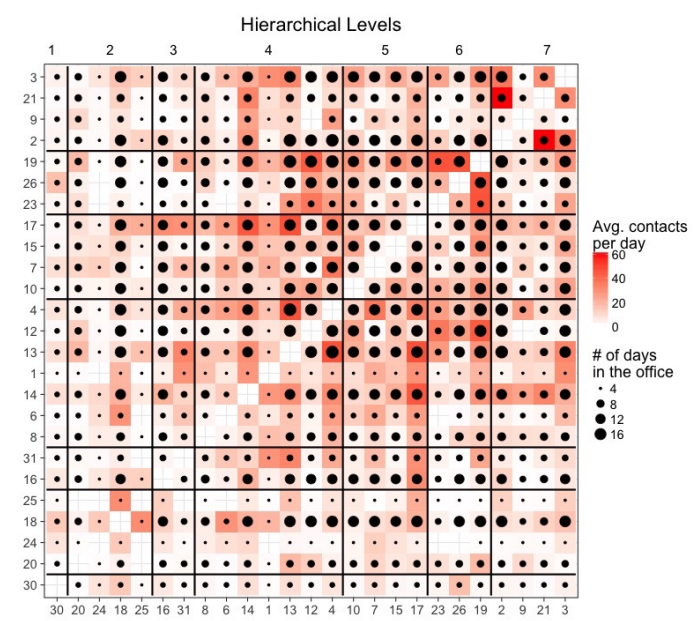

(b) Participants sorted by their level in the organizational structure.

Fig. 4. Normalized number of contacts for each pair of participants for the entire study. The horizontal and vertical lines separate the participants in the different groups. The size of the circles overlaid represents the number of days that both participants in each pair were in the office at the same time.

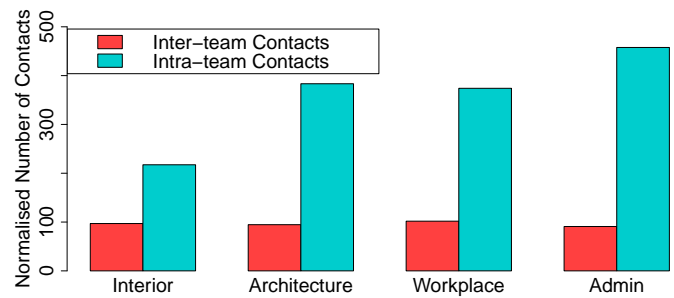

Fig. 5. Normalized number of inter and intra-team contacts for each of the larger teams.

contacts in Circulation and Kitchen might represent more spontaneous ones. Both are in fact highly integrated into the spatial system of the office and research shows that integrated spaces attract more activities [42]. The kitchen also acts as an attractor in line with previous findings highlighting the role of social spaces in fostering inter-team contacts [8].

The second aspect of the organizational structure that we analyse is the vertical division into hierarchical levels. Figure 4(b) shows the aggregated number of normalized contacts with the participants ordered by their level in the hierarchy. The main pattern that emerges is that there are fewer contacts among the upper levels (i.e. 1, 2 and 3) than among the lower levels. In fact, the plot shows darker and denser regions from level 4 to 7 going towards the upper right corner. We also notice that there are several pairs formed by a person from an high rank role (levels 1,2 or 3) and a person from a low rank role where, despite they were in the office for 
several days, the average number of contacts is low. Vice versa there are pairs with both people from low rank roles that were together in the office for few days but had more contacts, on average. This shows that even when high rank people are in the office, they have less contacts with others and this might be related to kind of work they have to do. To test the significance of the patterns we observe we first looked at the pairwise intra-level contacts within low (4, 5, 6 and 7) and high levels (1,2 and 3) and found a significant difference in the two distributions (Kruskal-Wallis rank sum test, $\mathrm{p}$-value $<0.05)$. We also found a significant difference ( $\mathrm{p}$-value $<0.01$ ) in the distributions of the pairwise inter-level contacts within low (4, 5, 6 and 7) and high levels (1,2 and 3). This supports the conclusion that participants in the lower levels have more contacts than the ones in the upper levels.

These results point to a strongly networked type of organization, where the way work gets done does not resemble the formal organizational hierarchy [31]. Instead, strong lateral links emerge among the lower ranks of the hierarchy across reporting lines and team affiliation. Burns and Stalker [9] have argued that this type of organization provides a suitable structure in dynamic organizational environments. It could be hypothesized that the $\mathrm{ABW}$ principles applied here do not hinder lateral communication and they might even facilitate it (given comparisons with reports of communication in non ABW studies [44]).

\subsection{Demise of Allocated Desks}

One of the central principles in $\mathrm{ABW}$ is the absence of allocated workstations. The assumption is that employees will change from one work location to another in order to best match the needs of the current task and personal preferences. In this section we use our methodology to understand to what extent this principle is implemented in the company we studied and if our participants adapted to this working style.

Figure 6 shows the Empirical Cumulative Distribution Functions of the number of distinct locations visited by each participant averaged per day, per week and the total for the entire duration of the study. We selected three thresholds on the dwell times to understand if there is a difference in the number of locations visited based on time spent at each location, this allowed us also to filter out very short dwell times that are due to people walking inside the office. Two-sided Kolmogorov-Smirnov tests (alpha $=0.05)$ performed on each pair of distributions, for each aggregation period (day, week and study), show that the differences among the distributions are significant (test statistic $\mathrm{D}$ ranging from 0.4 to 1 and p-value ranging from $2.778 \mathrm{e}-11$ to 0.03663$)^{1}$.

Looking at the distributions for dwell times larger than or equal to ten minutes we see that participants visit almost half of the monitored locations (13 locations excluding "Outside office") during an average day and almost all locations if we consider the entire study, suggesting a great level of mobility in the office. However, when we consider longer dwell times, participants explore significantly fewer locations. In particular, Figure 6(a) shows that on an average day the employees visit slightly more than one location for one hour or more, meaning that people rarely use more than one location per day for long tasks. Looking closer at the data for dwell times equal or longer than 1 hour, we observe that the maximum number of work locations used in any day of the study is 2 and $52 \%$ of the participants worked at least once in 2 different locations in any day of the study. However, when we aggregate the data per week, these figures rise to a maximum of 4 distinct locations in any week of the study and $80 \%$ of the participants worked at least once in 2 or more distinct locations in any week. We point out that only two employees, belonging to the Administration team, have assigned desks.

To understand which are the locations used to carry out longer tasks we computed the average dwell time per location (Figure 7(a)). We observe that the locations with the longest average dwell times are also the biggest workstations on the two floors (workstation 1 and 13) and two of three non-private workstations that have computers for the employees. We further observe that private workstations number 6 and 16 present also long dwell times probably for individual and focused work. By contrast, the meeting room number 10 seem less used than the others probably because it does not have doors and therefore it would be difficult to have a meeting in

\footnotetext{
${ }^{1}$ We performed the Kolmogorov-Smirnov test because it does not assume a specific underlying distribution.
} 


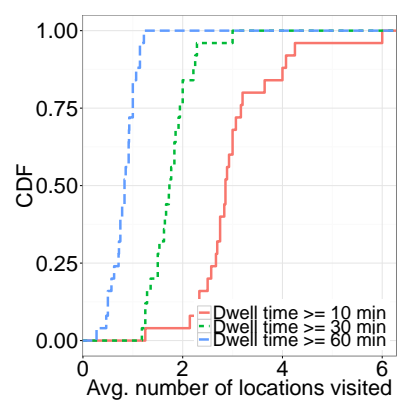

(a) Average number of locations visited per day.

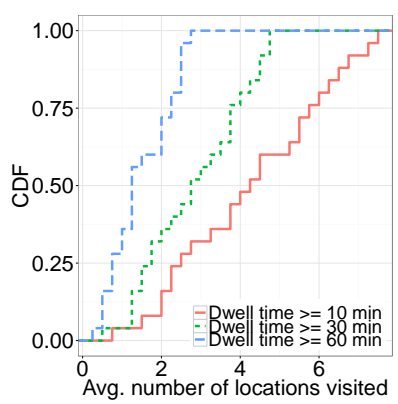

(b) Average number of locations visited per week.

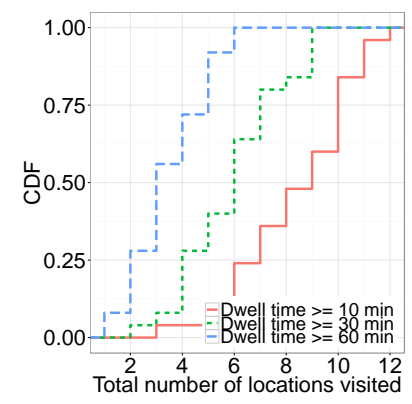

(c) Total number of locations visited in the entire study.

Fig. 6. Empirical Cumulative Distribution Functions of the number of locations visited by each participant averaged per day, averaged per week and the total number for the entire study for different dwell time thresholds. The "Outside office" location is excluded because not every employee is required to carry out work tasks outside.

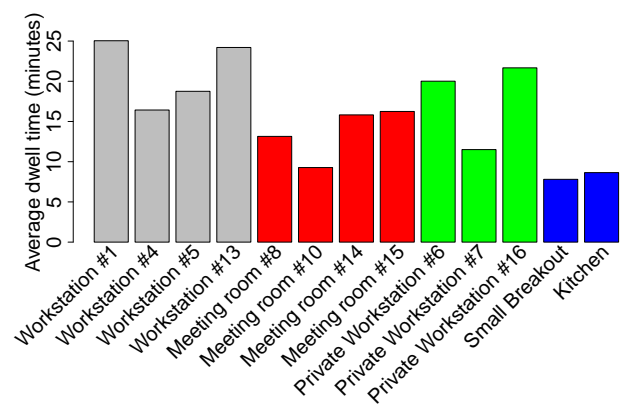

(a)

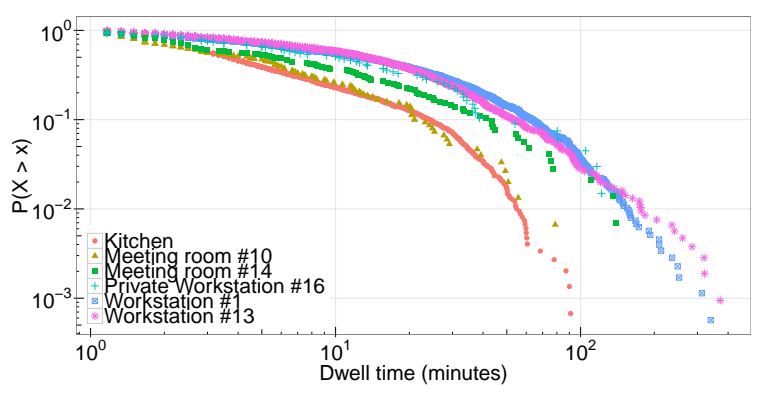

(b)

Fig. 7. Analizing dwell times for different locations inside the office. (a) shows the average dwell times for each location. The "Outside office" location is excluded because not every employee is required to carry out work tasks outside. (b) shows the Complementary Cumulative Distribution Function (CCDF) of dwell times of certain locations representative of all the room categories considered (log-log plot).

isolation from the rest of the office. The kitchen also seems used for short periods of time. Figure 7(b) shows the distributions of certain locations that are representative of the room types we considered. It is possible to observe that open space and private workstations have longer tails and are used for longer periods while the kitchen and the meeting room \#10 have different distributions and host people for shorter periods of time.

These results suggest that the employees might not have completely adopted the ABW principles. In fact, switching setting within a day, for work related tasks ( $>=60$ minutes), is not very prevalent. Similar results have been reported by Appel-Meulenbroek et al. where $68 \%$ of the employees surveyed never switches during an average day and only $14 \%$ switches once [4]. However, if we consider a weekly time scale we can see that choosing different settings is more likely. This behaviour is also highlighted by the employees' mobility self-assessment (see "Workplace Deployment" section) where they reported greater level of mobility. So, while at a very fine-grained temporal scale $\mathrm{ABW}$ traits are not observed, they are indeed observed at a coarser temporal granularity, retaining 


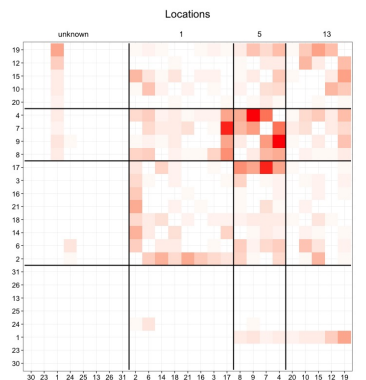

(a) Locations day 1

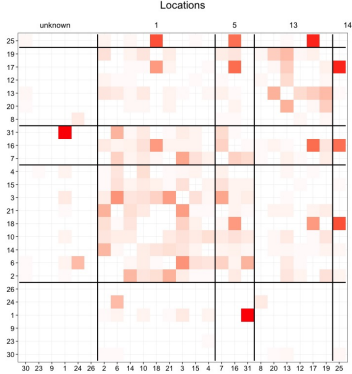

(b) Locations day 2 .

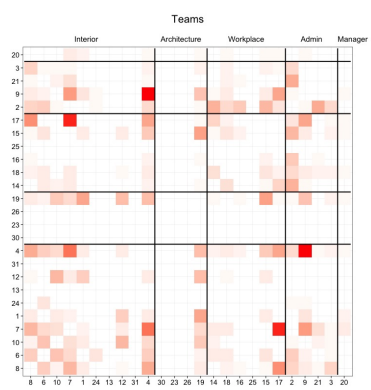

(c) Teams day 1

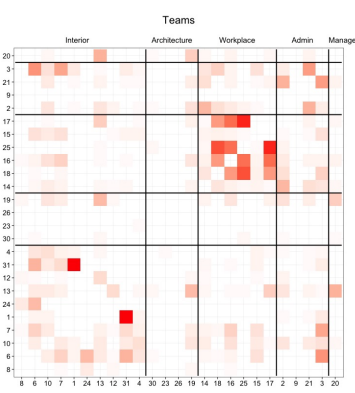

(d) Teams day 2 .

Fig. 8. Number of contacts for each pair of participants for two days of the study. The contacts happened at the main workstations have been omitted. In (a) and (b) the participants are sorted by the location they chose for the day while in (c) and (d) they are sorted by the team they belong to. The darker the colour the more contacts between the people. The solid horizontal and vertical lines separate the participants in the different groups. The location "unknown" represents participants that were not in the office that day or for whom we do not have location information (device malfunction).

the advantages related to serendipitous encounters and potential idea exchanges which are usually associated to this $[10,43]$.

\subsection{Social Ties and Agile Working}

Given the previous results where we showed that people tend to use on average one work location for long tasks we try now to understand if this could be due to the fact that people work in teams. Towards this objective we first looked at the contacts for each day of the study. Figure 8 reports the netgraphs for two representative days as an example. The patterns for the other days are highly correlated with the ones we show here. Each square represents the number of contacts between a pair of people and a darker colour means more contacts. On the left the participants on the two axis are sorted by the locations they chose for the day while on the right by the team they belong to. Given that our devices capture only proximity, it is obvious that working at the same location increases contact intensity, therefore we intentionally removed all the contacts that happened at the main desks (1, 4, 5 and 13) and considered only contacts detected somewhere else in the office.

From the plots it is visible that there are more contacts among people sitting at the same workstation rather than people in the same team. In fact, the diagonal (from bottom-left to top-right), which represents pairs sitting at the same location or in the same team, has denser and darker colours in the plots on the left. By contrast, the plots on the right show a sparser and less defined pattern indicating that there are several contacts across different teams. A Kruskal-Wallis rank sum test [33] of the number of intra-team contacts and intra-location contacts (between people who chose the same desk for the day) for each day of the study shows that the differences among the distributions are significant ( $\mathrm{p}$-value $<2.2 \mathrm{e}-16)^{2}$. As reported in Figure 8 the contacts detected at the main workstations (1, 4, 5 and 13) have been removed.

To further study this difference we divided all the possible pairs of participants in two groups:

- Strong Ties: pairs that have a number of contacts greater than or equal to the average of the contacts happened away from the main workstations (1, 4, 5 and 13);

- Weak Ties: pairs that have a number of contacts less than the average of the contacts happened away from the main workstations (1, 4, 5 and 13).

\footnotetext{
${ }^{2}$ We performed a Kruskal-Wallis test because the data is not normally distributed, therefore we had to adopt a non-parametric test.
} 


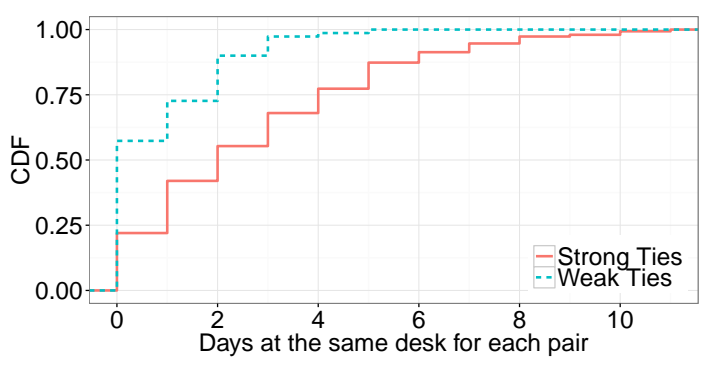

Fig. 9. Empirical Cumulative Distribution Function of the number of days pairs of participants choose to stay at the same desk for the Strong and Weak Ties groups.

Table 4. Characteristics of the data sets.

\begin{tabular}{cccc} 
Dataset & Participants & Study Period & Contacts \\
\hline Spacelab & 25 & 10 days & 2190 \\
Brown Old & 39 & 10 days & 683 \\
Brown New & 48 & 10 days & 1065 \\
\hline
\end{tabular}

For all pairs of participants we also determined the total number of days in which they chose the same desk. We found that the pairs that have strong ties, on average, choose to stay at the same desk more than the pairs that have weak ties. Strong ties in fact stay on average 2.682 days $($ median $=2)$ at the same desk while the weak ties only 0.819 days (median $=0$ ). Figure 9 shows the Empirical Cumulative Distribution Function of the number of days the two groups of pairs spent at the same desks. Clearly the pairs in the strong ties group prefer to stay at the same desk more than the people in the weak ties group. A Kruskal-Wallis rank sum test [33] shows that we can reject the null hypothesis that these two samples are drawn from populations with the same median values (p-value $<2.2 \mathrm{e}-16)^{2}$. An analysis of the teams of the participants revealed that $33.77 \%$ of the pairs in the strong ties group are made of people from the same team while for the weak ties only $14.09 \%$ of pairs. This suggests that some of the strong ties might be due to the fact that people work together in the same team and this might be one of the explanations why they choose the same desk more often. However, we cannot speak of causality. This result also highlights how intensely spatial, behavioural and organizational phenomena are entangled.

In summary, our results here suggest a mismatch between ABW principles and the actual use of space. In particular, the principle of allowing people free movement and choice of work location has not come to full fruition in the context studied. Pairs with high co-presence in locations away from desks (strong ties) also stick together at the same workstations much more than pairs with weaker ties. Team affiliation played a strong role in this. In short, people stick together with those they like or work with and choose their location not simply based on their task or the appropriate spatial setting for the day but with a social focus in mind. Relationships seemed to matter. Whether this process worked based on attraction (seeking like-minded people for one's close proximity) or repulsion (avoiding those one does not like) would require further research. Either way, social clusters were formed based on preferences and thus resisted the randomizing effect $\mathrm{ABW}$ wishes to have in an ideal world. 


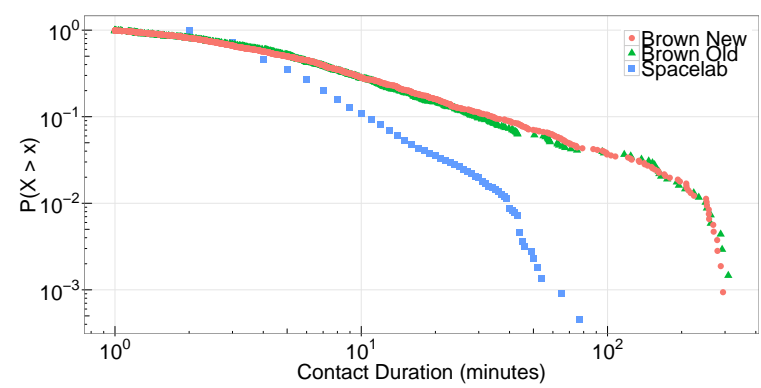

(a) CCDF of contacts duration (log-log).

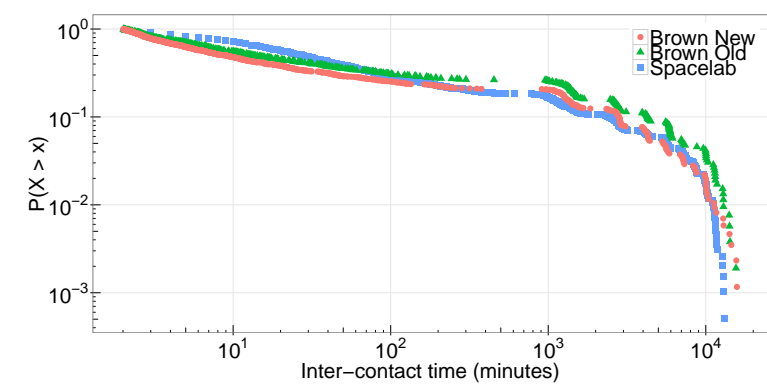

(b) CCDF of inter-contact times (log-log).

Fig. 10. Complementary Cumulative Distribution Functions of contact durations and inter-contact times.
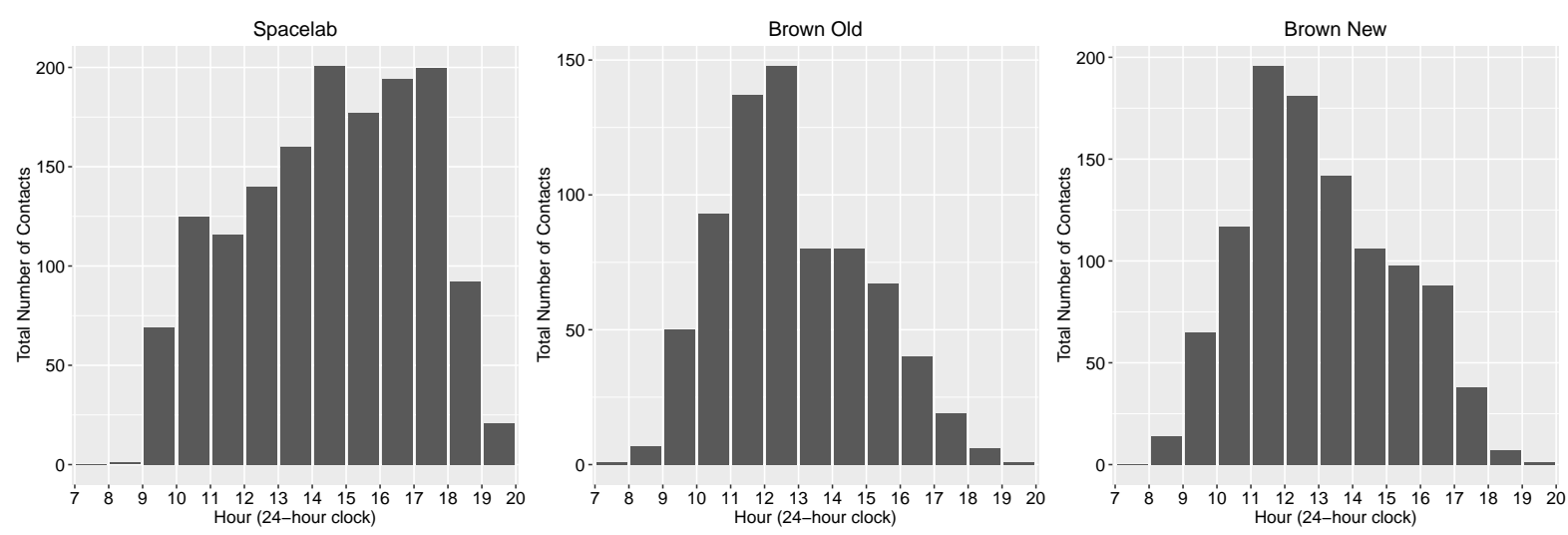

Fig. 11. Total number of contacts aggregated in 60-minute windows by hour of the day.

\subsection{Comparison with Traditional Offices}

In this section we aim to better understand the impact of ABW's principles on social dynamics inside the company by comparing our data set with two other data sets collected with the SocioPatterns badges [11] in two different offices [8]. SocioPatterns badges, similarly to our BLE devices, use radio beacons to detect close encounters.

The two data sets have been collected by Brown et al. in 2012 and 2013 in a research institution in UK that moved from one building to another during the study period (Table 4 ). The new building was designed for that specific research institution with the main aims of increasing the chances of serendipitous encounters among the employees and motivate an increased use of shared spaces. This was achieved by placing a central cafeteria on the ground floor and including larger lab spaces and more open areas. The old building represents an example of a traditional office with individual offices and few open or shared areas. The new building instead is closer to the $\mathrm{ABW}$ principles where diverse settings are offered to people for different kind of activities. However, in both buildings the employees had assigned desks and this might significantly impact the contact patterns we observe compared to our deployment at Spacelab.

We first analyze the contact durations and inter-contact times distributions shown in Figure 10. We observe that for the two buildings studied by Brown et al. the distributions have longer tails and longer contacts have been recorded compared to our deployment at Spacelab. This might suggest a successful implementation of ABW 

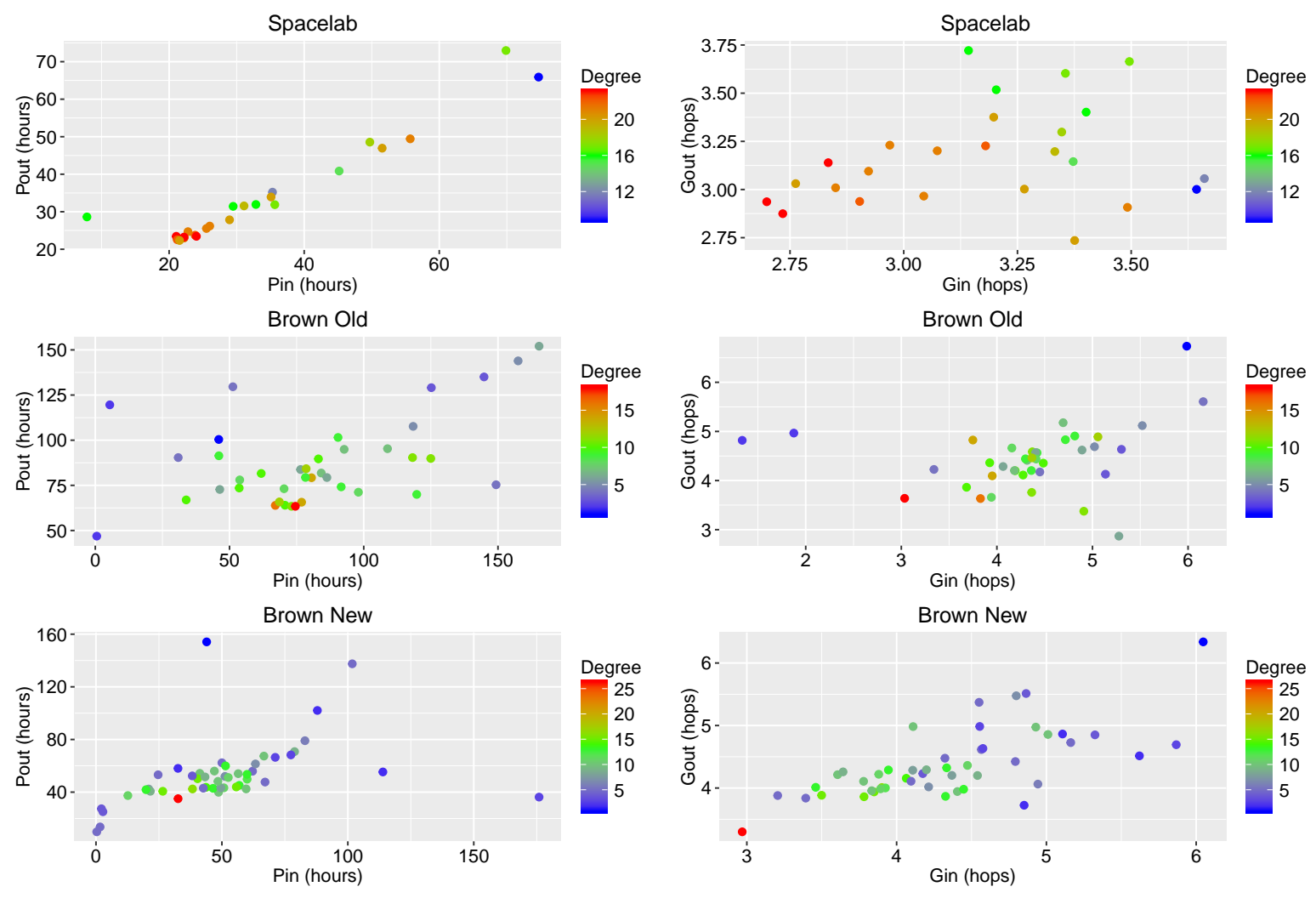

Fig. 12. Correlation between $P_{\text {in }}-P_{\text {out }}$ and $G_{\text {in }}-G_{\text {out }}$ for the three data sets. Each point represents a participant and is coloured according to the vertex degree calculated on the aggregated networks.

principles at Spacelab where employees tend to have more frequent but shorter interactions. However, this could also be a reflection of the nature of work done in the different organizations. On the one hand, Spacelab is a young company with a very dynamic and flexible working style while, on the other hand, the research institution might have a more traditional working style. For what concerns the inter-contact times instead we observe similar distributions for the three data sets.

Taking advantage of the fact that the three data sets have temporal information, we plot in Figure 11 the total number of contacts during different hours of the day for the entire duration of the study. We notice that, while in the two data sets collected by Brown et al. there is a visible pattern where the number of contacts increases around midday, in the Spacelab data set we see less regularity in the number of contacts over time and more interactive afternoons than mornings. We also observe how there are, on average, more contacts at Spacelab than in the other two offices. We hypothesize that the differences we observe are due to the application of ABW principles at Spacelab and this results in a more even distribution of contacts across the day, opposed to a more structured pattern in the other company, where people primarily stay in their office, having most of the contacts during lunch time. However, there are many other factors that could contribute to this behaviour and therefore we cannot speak of causality. Nevertheless, it is interesting to note that the office change observed in Brown et al. resulted in an increased number of contacts but it did not have changed the overall shape of the distribution of 
contacts during the day. We can only speculate that the additional contacts are an effect of the purposely built office and that a full adoption of ABW principles could possibly lead to a more substantial change in the daily patterns.

To further study the temporal characteristics of the data sets we borrow the metrics Average temporal proximity $(P(X, Y))$ and Average geodesic proximity $(G(X, Y))$ from [30]. The metrics measure the average time needed to to go from vertex $\mathrm{X}$ to vertex $\mathrm{Y}$ and the average number of hops between $\mathrm{X}$ and $\mathrm{Y}$ respectively. The temporal proximity considers edge availability over time and takes into account possible wait times at one vertex before moving to the next one. The geodesic proximity instead counts only the number of hops from one vertex to another (without considering the time needed for the hop) but it is still subject to the temporal restrictions in the network. Kostakos also defines $P_{\text {in }}(X)$ and $P_{\text {out }}(X)$ as measures of "how quickly, on average, $\mathrm{X}$ is reached by the rest of the network" and "how quickly, on average, $X$ reaches the rest of the network". Similarly, $G_{\text {in }}(X)$ and $G_{\text {out }}(X)$ are defined as "the average number of hops needed to reach X from the rest of the network" and "the average number of hops needed to reach the rest of the network from X".

In Figure 12 we plot the correlation between the in and out components of temporal proximity and geodesic proximity colour coding each participant according to the number of its connections in the aggregated network. Above all we observe that people at Spacelab reach the rest of the network and are reached in less time compared to the other two offices. Both in terms of time and number of hops. This could be attributed to the absence of assigned desks which brings employees into contact with a larger and more diverse set of people. Similarly, the presence of a central cafeteria in the new building studied by Brown et al. might be responsible of the reduced time needed to reach the network and being reached that we observe when comparing the old and new building.

It is also interesting that the relation between $P_{\text {in }}$ and $P_{\text {out }}$ is more structured for Spacelab than for the other offices where there is more variability among the participants. The old and new office both presents locally low-connected people that are quick at reaching the network or being reached by the network (blue points towards the bottom left corner) while for Spacelab the variation between people is much smaller.

These results give an indication, from a temporal perspective, of the possible effects Activity-Based Working has in the workplace. Obviously, we cannot speak of causality since there are several other factors that play an important role, such as: the type and structure of the organization and the kind of work done, the culture inside the office and the personality of individuals.

\section{DISCUSSION}

In this work we have analysed the fundamental ABW's principles that are common to implementations of the concept (flexible use of office space and collaboration opportunities). We have shown how technology can be used to collect data about human behaviour in a dynamic workplace and to allow reflection on how much ABW's principles have been absorbed by the office settings. Gathering suitable data is usually very difficult in environments where behaviour tends to vary, change and evolve more than in traditional settings. This makes our technology based solution even more key to these sort of validation studies. In the following we discuss our results with regard to implications for designers of $A B W$ office spaces.

\subsection{Theoretical and Practical Implications}

From the collected data, we found that the different teams in the company present a considerable level of intergroup contacts which might be indicative of high collaboration. A different pattern instead can be observed for the vertical structure. In fact, higher levels of co-presence are visible among people at the lower levels of the hierarchy. These results show that ABW principles were realized to some degree: the aim of allowing interaction and collaboration to spread across team boundaries seems well achieved. The high level of inter-group contacts speaks of an equal, almost random spreading of contacts across the organization as a whole. Together with an 
open plan layout that connects both floors visually, the spatial layout in conjunction with agile working provides ample opportunities for co-presence. This allows communication to flow vertically along teams and reporting lines, and on the lower hierarchy levels (among people with more time available) provides social glue and creates a "networked" organization. Similar results have been observed also in other ABW offices where employees reported greater satisfaction for informal un-planned meetings, informal social interaction and collaboration on creative and focused work [34]. Regarding the analysis of the vertical structure, to our knowledge, only one previous work analysed, using wearable sensors, its effect on social contacts in a research facility without ABW. The authors reported no significant impact of the management structure on social connectivity [8]. This confirms the need to study these dynamics in various settings to better understand the generalizability of the findings.

For what concerns mobility inside the office, we discover that the ABW principle of not having allocated desks might not be well received by the employees, at least when a fine-grained temporal scale is considered. Our results show that desk selection seems to be constrained by strong team-related social clusters. On an average day in fact, employees explore various locations for short periods but rarely change settings when longer dwell times are considered. However, more mobility is observed when we consider an average week. Additionally, we discover that pairs of people with more contacts (strong ties) tend to choose nearby desks more often than people with less contacts (weak ties). This suggests the hypothesis that employees are driven by the presence of specific colleagues when choosing the work setting. Similar results have already been observed in other ABW environments but have never been related to proximity contacts as we do in this work. Leesman reported that, even within ABW offices, large numbers of employees fail to adopt Activity-Based behaviours and have rather limited mobility dynamics [34]. Also Appel-Meulenbroek et al. uncovered misuses concerning office areas, and found that most of the employees use up to 2 different types of space and never switches during an average day [4]. This represents a challenge for architects and office managers that have to deal with what it seems opposition to change by employees and habitually driven working styles. A possible solution could lie in the involvement of users in the design process with the objective to adapt ABW principles and implementations to users's needs and preferences. Additionally, training sessions might be useful to clarify the benefits of more mobile behaviours. This also raises interesting questions for future research. Efforts could be directed towards the understanding whether this behaviour is driven by attraction (seeking like-minded people for one's close proximity) or by repulsion (avoiding those one does not like) and if the choice of setting, and so who to sit next to, adds to satisfaction and increases happiness at work or not.

Our work has also practical implications for the future design of activity-based workplaces. The results presented here are indeed insightful, as they show the powerful effect of allowing employees completely free choice of where to sit. The company we studied, had a good level of inter-team contact and a significantly higher number of co-presence events between participants in the lower ranks of the hierarchy. Previous research has shown that temporary co-location can increase the possibility of collaboration between scientists [6]. This could be used as guidelines in evidence-based design to encourage organizations who wish to become more collaborative and leverage knowledge-sharing across teams by introducing proximity to a wider range of people. Specifically, against the background that often organizations moving towards Activity-Based Working do not allow completely free choice of desks, but assign certain areas to certain teams, which could potentially limit the benefits of widespread contact patterns. However, our results have also shown that the switching between work locations and desk selection seems to be driven by team-related and social preferences, so in effect there might be less randomization of contact than appears at first sight. Clearly more research of different settings would be required to establish a clearer relationship between the amount of choice in agile working and the degree of dynamic contact and co-presence. Additionally, it would be interesting to explore the possibility of voluntarily disrupt people habits (i.e., sitting always at the same location) to understand if it would be beneficial and in which way. 


\subsection{Drawbacks of ABW Concepts}

In this work we have seen that the participants did not completely adapted to the ABW principles, especially when looking at the mobility and sitting arrangement patterns. Our main objective was to uncover emerging office dynamics from a quantitative point of view, hence the study of why participants preferred certain behaviours is beyond the scope of this paper and certainly relevant for future work. Here we discuss what other works have found in this area and relate back to our results. De Been et al. studied employees satisfaction in $20 \mathrm{ABW}$ environments with questionnaires and group interviews [18]. The open layout of the work environment has been acknowledged to stimulate more communication between different departments and to increase knowledge sharing. This is what we found at Spacelab where inter-group contacts seem to be quite substantial and where dynamics are different than traditional offices. Nevertheless, De Been et al. reported that the office layout also had negative effects, finding that people experienced lack of possibilities to concentrate, lack of privacy and unavailability of desired work points (i.e. waste of time finding a desk, attractive ones already occupied), with the latter mentioned also by Appel-Meulenbroek et al. [4]. However, we can argue that the lack of places for concentration and privacy could also apply to open plan offices with fixed seating allocation, so they not necessarily relate to ABW exclusively.

In another study, De Been et al. compared different office types including flexible settings (ABW) [17]. They conclude that $\mathrm{ABW}$ environments do not support productivity, privacy and concentration as well as enclosed or open offices (with fixed desks). The authors provide one possible explanation for the difference between assigned and unassigned desks in terms of psychological identity. In ABW offices people are not assigned to specific workstations and they cannot personalise the spaces they use. This might result in people not feeling attached to their workplace anymore and therefore having a lower satisfaction. The study also mentions the difficulty of finding people as a potential drawback, which could explain why ABW offices are rated lower with regards to satisfaction with communication. In the company we studied this is less of a problem since the office is small enough. However, usually, in larger settings ABW is often arranged with team zones and there is a dedicated area to go to, if one is looking for somebody, meaning that people can be found, if needed.

\subsection{Technology Considerations}

We have shown how sensing could be used to gather spatio-temporal information in the workplace. This information can easily create the foundation for ubiquitous applications. For example, it can be made available to the employees as new data along the line of the "quantified self" movement. This feedback could potentially encourage people to seek more informal contacts with others they usually do not interact with, as a way to increase productivity and generate new ideas [29]. The location information could be useful to space management representatives to optimize space usage based on objective fine-grained data and not only on feelings or sparse surveys. Finally the applications of this technology are definitely not limited to workplaces, other settings, such as large events, could also benefit from it.

Large deployments pose other challenges and issues. First of all, to cover a large office space, possibly split over multiple buildings, more localisation static beacons have to be deployed and maintained. One possibility in this situation would be to use the existing WiFi and BLE infrastructure to locate participants and deploy dedicated beacons only in specific areas where more resolution is necessary or where WiFi/BLE coverage is not optimal. In terms of mobile devices, one potential issue regards the possibility of radio signals collisions that might prevent correct detection when many devices are in range. However, this is likely to be a real problem only during large events where many employees would attend and can be avoided by correctly tuning the transmission frequency to reduce collision probability. More realistic is the challenge of adoption. In fact, the burden of carrying an additional device might be too big for employees and this could result in limited adoption inside the company. In case a dedicated device has to be used, conceivably because special sensors have to be employed, it is important to 
pay particular attention to its comfort and ease of use, especially if the study is planned to run for a long period. In particular, the battery life is one of the most important aspects. The device should be built and tuned to allow the data collection at a reasonable rate without disturbing the user with the need for frequent charges. In alternative, it would be possible to use devices that people already carry with them (e.g. smartphones and smartwatches) even if this might result in limited data resolution and accuracy. Additionally, if smartphones are used another aspect to consider is the fact that people do not always carry their phone when indoor, therefore alternative strategies have to be devised (e.g. use the smartwatch when the phone is not with the person). Moreover, if long deployments are planned it is crucial to engage the participants with the study and data collection by, for example, providing statistics about the study or make the devices useful beyond data collection (e.g. the device could double as access card). This should motivate people to use the device resulting in more quality data.

We acknowledge that the particular technology we used for this work (BLE wearable devices) is not capable of providing an exact detection of social contacts. Our devices include a larger range of contacts, compared for example to participant observations, because they record every time people are in close physical proximity even if they are not engaged in a conversation. Our findings suggest that although it does not capture the exact type of interaction (i.e., it is not possible to know if two people were actually talking or not), it provides usable information to aid the study of behavioural patterns and dynamics in the workplace. Other technologies, such as the Sociometric badges [14], provide more directional information about face-to-face contacts hence might offer a finer grained view. However, even with this approach there are drawbacks. For example, the system might be more sensitive to false negatives in cases where people are side-by-side or in large groups with more distance between the participants. Accurate social interaction monitoring is still an hard problem especially because there are different aspects that can be captured (e.g., proximity, distance, angle of contact, communication and content). Current and past devices usually focused on one or few of these aspects so the collected data will always represent an approximation. In our specific deployment we opted for the least intrusive method that could give us enough information to study emerging ABW patterns.

\subsection{Limitations}

Generalization. Different companies, with distinct organizational structures and cultures might present contrasting interaction patterns which might lead to different conclusions to the ones presented here. The company we considered is an architecture firm and working style might be different in organizations operating in other sectors (e.g., commercial or scientific) or on different continents and with different cultures. More studies are needed to capture and understand dynamics that could be generalized more widely. The methodology proposed however is applicable to other organizations, facilitating the validation of ABW principles despite the spatio-temporal challenges of the office dynamics.

Scalability. Bigger companies with more employees might show different interaction patterns: there might be inertia in interacting outside teams, unlike in our limited setting. Moreover, dynamics of team formation when people join or leave should be studied at a longer time scale. Effects of space and social ties on productivity also needs longitudinal efforts.

Technology Accuracy. Our devices are able to record proximity contacts and not actual communication. Therefore it is possible that our data overestimates the actual contacts because our logs represent potential communication opportunities rather than real communication events. Similarly, for the locations traces, some dwell times might be underestimated as it is possible that, even if a person is always sitting at the same location, the device could detect radio signals from other beacons and temporarily associate the person to that beacon. Nevertheless, for social psychology theories, physical proximity increases chances of interaction among people [23].

Comparison Validity. When comparing data collected in different buildings, organisation-specific variables, such as, structure of the organisation, its culture and people personality, might affect the validity of comparisons. 
However, by showing a comparative analysis with traditional offices we are able to gain insights on how ABW's principles might affect social dynamics even if additional research is needed to better generalise the results. Likewise, the technology used to collect the data was slightly different: however this should not compromise the results as we have compared the data from the two technologies and found that they have overall similar properties [39].

\section{CONCLUSION}

We have shown how data from wearables can be used to offer insights about the adoption of ABW principles in companies trying to adopt them. The same setting would be very challenging to study with traditional methods of observations. Our work offers a mechanism for space designers to reflect on the application of ABW principles and study its impact longitudinally.

Our future work includes additional deployments to understand the potential for generalization of our findings to other dynamic company environments and the inclusion of further sensors to increasing accuracy of interaction estimation. We also would like to run longer longitudinal studies to analyse productivity and team formation processes in more detail.

\section{ACKNOWLEDGMENTS}

Alessandro Montanari acknowledges the support of the EPSRC and Qualcomm Inc. through a Research Studentship in Computing. The authors thank Spacelab employees for their support and participation in the user study and Efstathia Kostopoulou for the participant observations.

\section{REFERENCES}

[1] Nadav Aharony, Wei Pan, Cory Ip, Inas Khayal, and Alex Pentland. 2011. Social fMRI: Investigating and shaping social mechanisms in the real world. Pervasive and Mobile Computing 7, 6 (2011), 643-659.

[2] Thomas J Allen. 1984. Managing the Flow of Technology: Technology Transfer and the Dissemination of Technological Information Within the R\&D Organization, volume 1 of MIT Press Books. (1984).

[3] Thomas John Allen and Günter Henn. 2006. The Organization and Architecture of Innovation: Managing the Flow of Technology. Routledge.

[4] Rianne Appel-Meulenbroek, Rianne Appel-Meulenbroek, Peter Groenen, and Ingrid Janssen. 2011. An end-user's perspective on activity-based office concepts. Journal of Corporate Real Estate 13, 2 (2011), 122-135.

[5] Tjeerd W Boonstra, Mark E Larsen, and Helen Christensen. 2015. Mapping dynamic social networks in real life using participants' own smartphones. Heliyon 1, 3 (2015), e00037.

[6] Kevin Boudreau, Ina Ganguli Prokopovych, Patrick Gaule, Eva Catharina Guinan, and Karim R Lakhani. 2012. Colocation and scientific collaboration: evidence from a field experiment. Harvard Business School Working Paper (2012).

[7] Chloë Brown, Christos Efstratiou, Ilias Leontiadis, Daniele Quercia, and Cecilia Mascolo. 2014. Tracking Serendipitous Interactions: How Individual Cultures Shape the Office. In Proceedings of the 17th ACM Conference on Computer Supported Cooperative Work \&\#38; Social Computing (CSCW'14). New York, NY, USA, 1072-1081. https://doi.org/10.1145/2531602.2531641

[8] Chloë Brown, Christos Efstratiou, Ilias Leontiadis, Daniele Quercia, Cecilia Mascolo, James Scott, and Peter Key. 2014. The Architecture of Innovation: Tracking Face-to-face Interactions with Ubicomp Technologies. In Proceedings of the 2014 ACM International foint Conference on Pervasive and Ubiquitous Computing (UbiComp '14). ACM, New York, NY, USA, 811-822. https://doi.org/10.1145/2632048.2632056

[9] Tom E Burns and George Macpherson Stalker. 1961. The management of innovation. University of Illinois at Urbana-Champaign's Academy for Entrepreneurial Leadership Historical Research Reference in Entrepreneurship (1961).

[10] Ronald S Burt. 2004. Structural holes and good ideas. American journal of sociology 110, 2 (2004), 349-399.

[11] Ciro Cattuto, Wouter Van den Broeck, Alain Barrat, Vittoria Colizza, Jean-François Pinton, and Alessandro Vespignani. 2010. Dynamics of Person-to-Person Interactions from Distributed RFID Sensor Networks. PLoS ONE 5, 7 (07 2010), e11596. https://doi.org/10.1371/ journal.pone.0011596

[12] Nitesh V. Chawla, Kevin W. Bowyer, Lawrence O. Hall, and W. Philip Kegelmeyer. 2002. SMOTE: synthetic minority over-sampling technique. Fournal of artificial intelligence research (2002), 321-357.

[13] Jinhyuk Choi, Seongkook Heo, Jaehyun Han, Geehyuk Lee, and Junehwa Song. 2013. Mining social relationship types in an organization using communication patterns. In Proceedings of the 2013 conference on Computer supported cooperative work. ACM, 295-302. 
[14] Tanzeem Choudhury and Alex Pentland. 2002. The sociometer: A wearable device for understanding human networks. In Proceedings of the CSCW'02 Workshop: Ad hoc Communications and Collaboration in Ubiquitous Computing Environments.

[15] Aaron Clauset and Nathan Eagle. 2012. Persistence and periodicity in a dynamic proximity network. arXiv preprint arXiv:1211.7343 (2012).

[16] Christina Bodin Danielsson and Lennart Bodin. 2008. Office type in relation to health, well-being, and job satisfaction among employees. Environment and Behavior 40, 5 (2008), 636-668.

[17] Iris De Been and Marion Beijer. 2014. The influence of office type on satisfaction and perceived productivity support. fournal of Facilities Management 12, 2 (2014).

[18] Iris De Been, Marion Beijer, and Dorieke Den Hollander. 2015. How to cope with dilemmas in activity based work environments-results from user-centred research. In Conference paper 14th EuroFM Research Symposium. EuroFM research papers.

[19] Trinh Minh Tri Do and Daniel Gatica-Perez. 2011. Groupus: Smartphone proximity data and human interaction type mining. In 2011 15th Annual International Symposium on Wearable Computers. IEEE, 21-28.

[20] Trinh Minh Tri Do, Kyriaki Kalimeri, Bruno Lepri, Fabio Pianesi, and Daniel Gatica-Perez. 2013. Inferring social activities with mobile sensor networks. In Proceedings of the 15th ACM on International conference on multimodal interaction. ACM.

[21] Nathan Eagle and Alex Sandy Pentland. 2006. Reality mining: sensing complex social systems. Personal and ubiquitous computing 10, 4 (2006).

[22] Mathieu Génois, Christian L Vestergaard, Julie Fournet, André Panisson, Isabelle Bonmarin, and Alain Barrat. 2015. Data on face-to-face contacts in an office building suggest a low-cost vaccination strategy based on community linkers. Network Science 3, 03 (2015), 326-347.

[23] Erving Goffman. 1961. Encounters: Two studies in the sociology of interaction. (1961).

[24] William Huang, Ye-Sheng Kuo, Pat Pannuto, and Prabal Dutta. 2014. Opo: a wearable sensor for capturing high-fidelity face-to-face interactions. In Proceedings of the 12th ACM Conference on Embedded Network Sensor Systems. ACM.

[25] Pan Hui, Augustin Chaintreau, James Scott, Richard Gass, Jon Crowcroft, and Christophe Diot. 2005. Pocket Switched Networks and Human Mobility in Conference Environments. In Proceedings of the 2005 ACM SIGCOMM Workshop on Delay-tolerant Networking (WDTN '05). ACM, New York, NY, USA, 244-251. https://doi.org/10.1145/1080139.1080142

[26] Maria Ianeva, Stéphanie Faure, Jennifer Theveniot, François Ribeyron, Cormac Crossan, Gilles Cordon, and Claude Gartiser. 2015. Pervasive Technologies for Smart Workplaces: A Workplace Efficiency Solution for Office Design and Building Management from an Occupier's Perspective. In Human Work Interaction Design. Work Analysis and Interaction Design Methods for Pervasive and Smart Workplaces. Springer, 73-82.

[27] Shuja Jamil, Anas Basalamah, and Ahmed Lbath. 2014. Crowdsensing traces using bluetooth low energy (BLE) proximity tags. In Proceedings of the 2014 ACM International foint Conference on Pervasive and Ubiquitous Computing: Adjunct Publication. ACM, 71-74.

[28] Shuja Jamil, Anas Basalamah, Ahmed Lbath, and Moustafa Youssef. 2015. Hybrid participatory sensing for analyzing group dynamics in the largest annual religious gathering. In Proceedings of the 2015 ACM International foint Conference on Pervasive and Ubiquitous Computing. ACM, 547-558.

[29] Phillip Jeffrey and Andrew McGrath. 2000. Sharing Serendipity in the Workplace. In Proceedings of the Third International Conference on Collaborative Virtual Environments (CVE' '00). ACM, New York, NY, USA, 173-179. https://doi.org/10.1145/351006.351037

[30] Vassilis Kostakos. 2009. Temporal graphs. Physica A: Statistical Mechanics and its Applications 388, 6 (2009), 1007-1023.

[31] David Krackhardt and Jeffrey Hanson. 1993. Informal Networks: The Company Behind the Chart. Harvard Business Review 71, 4 (jul/aug 1993), 104-111.

[32] David Krackhardt and Robert N Stern. 1988. Informal networks and organizational crises: An experimental simulation. Social psychology quarterly (1988), 123-140.

[33] William H Kruskal and W Allen Wallis. 1952. Use of ranks in one-criterion variance analysis. fournal of the American statistical Association 47, 260 (1952), 583-621.

[34] Leesman. 2016. Activity Based Working - The rise and rise of ABW: Reshaping the physical, virtual and behavioural workspace. (2016). Retrieved 03/08/2016 from http://leesmanindex.com/wp-content/uploads/Lees002-ABW-SP-INT-version.pdf

[35] Bruno Lepri, Jacopo Staiano, Giulio Rigato, Kyriaki Kalimeri, Ailbhe Finnerty, Fabio Pianesi, Nicu Sebe, and Alex Pentland. 2012. The sociometric badges corpus: A multilevel behavioral dataset for social behavior in complex organizations. In Privacy, Security, Risk and Trust (PASSAT), 2012 International Conference on and 2012 International Confernece on Social Computing (SocialCom). IEEE.

[36] Machine Learning Group at the University of Waikato. 1997. Weka 3: Data Mining Software in Java. (1997). Retrieved 09/12/2015 from http://www.cs.waikato.ac.nz/ml/weka/

[37] Afra Mashhadi, Utku Günay Acer, Aidan Boran, Philipp M. Scholl, Claudio Forlivesi, Geert Vanderhulst, and Fahim Kawsar. 2016. Exploring Space Syntax on Entrepreneurial Opportunities with Wi-Fi Analytics. In Proceedings of the 2016 ACM International foint Conference on Pervasive and Ubiquitous Computing (UbiComp '16). ACM, New York, NY, USA, 658-669. https://doi.org/10.1145/2971648. 2971745

[38] Eline M Meijer, Monique HW Frings-Dresen, and Judith K Sluiter. 2009. Effects of office innovation on office workers' health and performance. Ergonomics 52, 9 (2009), 1027-1038.

, Vol. 1, No. 1, Article 1. Publication date: February 2018. 
[39] Alessandro Montanari, Sarfraz Nawaz, Cecilia Mascolo, and Kerstin Sailer. 2017. A Study of Bluetooth Low Energy Performance for Human Proximity Detection in the Workplace. In Proceedings of the 2017 IEEE International Conference on Pervasive Computing and Communications (PerCom). IEEE.

[40] Daniel Olguín Olguín and Alex Sandy Pentland. 2008. Social sensors for automatic data collection. Proceedings of the 14th Americas Conference on Information Systems (AMCIS) (2008).

[41] Daniel Olguín Olguín, Benjamin N Waber, Taemie Kim, Akshay Mohan, Koji Ara, and Alex Pentland. 2009. Sensible organizations: Technology and methodology for automatically measuring organizational behavior. Systems, Man, and Cybernetics, Part B: Cybernetics, IEEE Transactions on 39, 1 (2009), 43-55.

[42] Alan Penn, Jake Desyllas, and Laura Vaughan. 1999. The space of innovation: interaction and communication in the work environment. Environment and Planning B: Planning and Design 26, 2 (1999), 193-218.

[43] A. Pentland. 2012. The new science of building great teams. Harvard Business Review 90, 4 (2012), 60-69.

[44] Kerstin Sailer. 2010. The space-organisation relationship. On the shape of the relationship between spatial configuration and collective organisational behaviours. Ph.D. Dissertation. TU Dresden, Germany.

[45] Kerstin Sailer and Ian McCulloh. 2012. Social networks and spatial configuration - How office layouts drive social interaction. Social networks 34, 1 (2012), 47-58.

[46] Kerstin Sailer, Ros Pomeroy, and Rosie Haslem. 2015. Data-driven design - Using data on human behaviour and spatial configuration to inform better workplace design. Corporate Real Estate fournal 4, 3 (2015), 249-262.

[47] Donald J Schuirmann. 1987. A comparison of the two one-sided tests procedure and the power approach for assessing the equivalence of average bioavailability. Journal of Pharmacokinetics and Pharmacodynamics 15, 6 (1987).

[48] Arkadiusz Stopczynski, Vedran Sekara, Piotr Sapiezynski, Andrea Cuttone, Mette My Madsen, Jakob Eg Larsen, and Sune Lehmann. 2014. Measuring large-scale social networks with high resolution. PloS one 9, 4 (2014), e95978.

[49] James B Stryker and Michael D Santoro. 2012. Facilitating face-to-face communication in high-tech teams. Research-Technology Management 55, 1 (2012), 51-56.

[50] Samuel Townsend, Mark E Larsen, Tjeerd W Boonstra, and Helen Christensen. 2015. Using Bluetooth Low Energy in smartphones to map social networks. arXiv preprint arXiv:1508.03938 (2015).

[51] Benjamin N Waber, Daniel Olguin Olguin, Taemie Kim, and Alex Pentland. 2010. Productivity through coffee breaks: Changing social networks by changing break structure. Available at SSRN 1586375 (2010).

[52] Frank Wilcoxon. 1945. Individual comparisons by ranking methods. Biometrics bulletin 1, 6 (1945).

[53] Christina Wohlers and Guido Hertel. 2016. Choosing where to work at work - towards a theoretical model of benefits and risks of activity-based flexible offices. Ergonomics (2016), 1-20. https://doi.org/10.1080/00140139.2016.1188220 arXiv:http://dx.doi.org/10.1080/00140139.2016.1188220

[54] Lynn Wu, Benjamin N Waber, Sinan Aral, Erik Brynjolfsson, and Alex Pentland. 2008. Mining face-to-face interaction networks using sociometric badges: Predicting productivity in an IT configuration task. Available at SSRN 1130251 (2008).

[55] Neil Zhao. 2010. Full-featured pedometer design realized with 3-Axis digital accelerometer. Analog Dialogue 44, 06 (2010), 1-5. 